\title{
Defectos

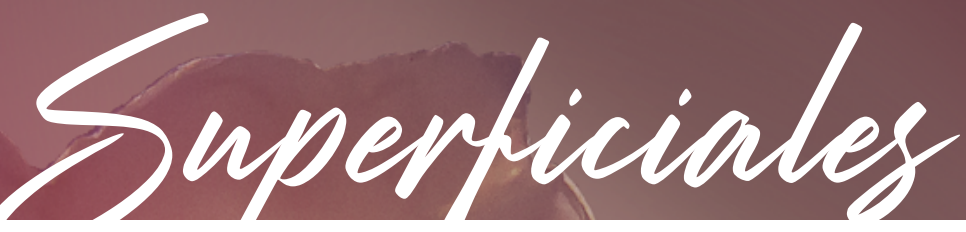

\section{de las limas mecanizadas de permeabilidad. Estudio in vitro}

"SURFACE DEFECTS OF MECHANIZED FILES OF PERMEABILITY USED IN MOLARS. IN VITRO STUDY"

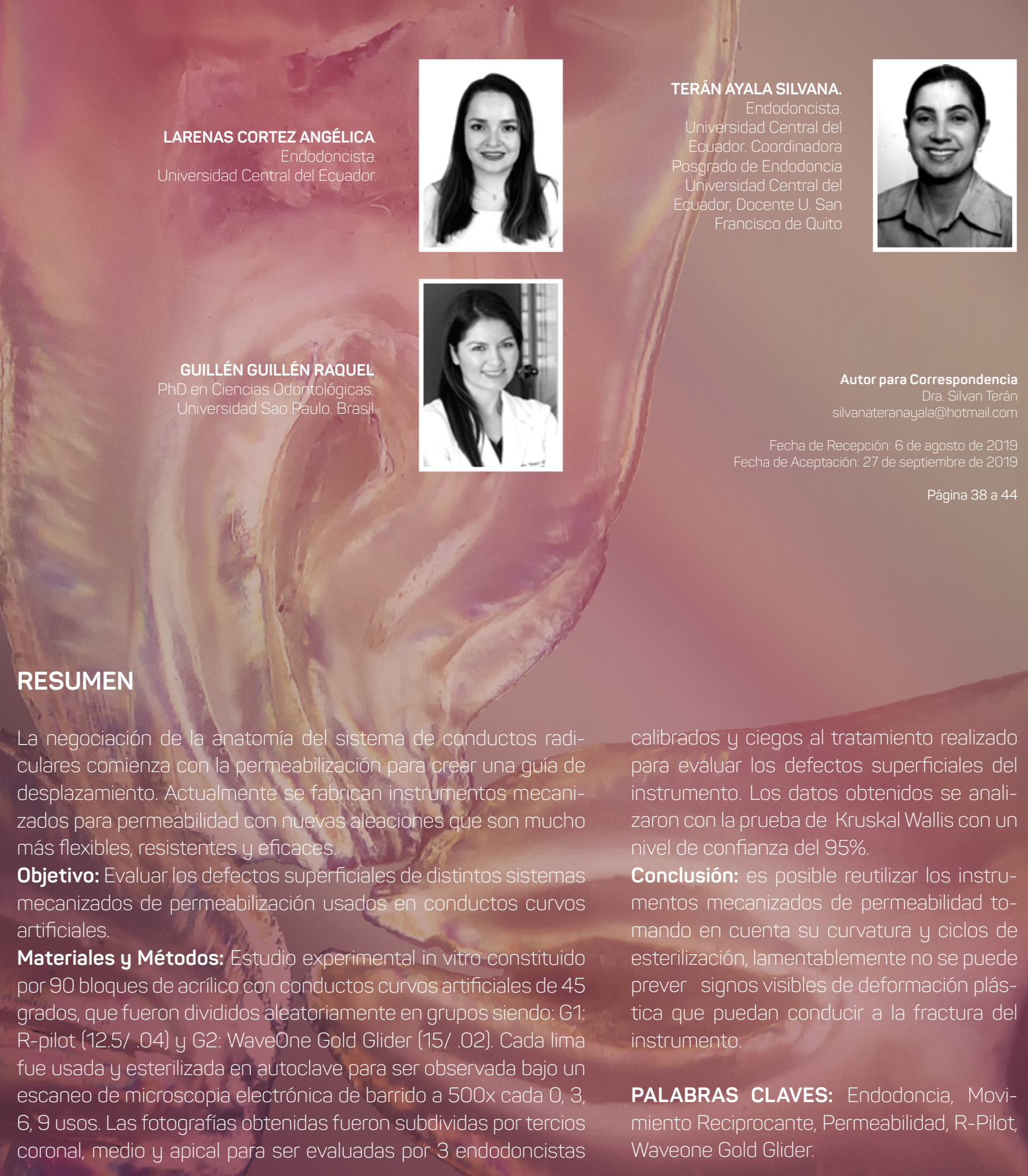




\section{SUMMARY}

Negotiation of the anatomy of the root canal system begins with permeabilization to create a displacement guide. Currently, mechanized instruments are manufactured for permeability with new alloys that are much more flexible, resistant and effective.

Objective: To evaluate the surface defects of different mechanized permeabilization systems used in artificial curved ducts.

Materials and methods: In vitro experimental study consisting of 90 acrylic blocks with 45 degree artificial curved ducts, which were randomly divided into groups: G1: R-pilot (12.5 / .04) and G2: WaveOne Gold Glider (15 / .02). Each file was used and sterilized in an autoclave to be observed under a scanning electron microscopy scanned at 500x every 0, 3, 6, 9 uses. The photographs obtained were subdivided by coronal, middle and apical thirds to be evaluated by 3 calibrated endodontists and blind to the treatment performed to evaluate the superficial defects of the instrument. The data obtained were analyzed with the Kruskal Wallis test with a 95\% confidence level.

Conclusion: It is possible to reuse mechanized permeability instruments taking into account their curvature and sterilization cycles, unfortunately no visible signs of plastic deformation that can lead to fracture of the instrument can be expected

Key words: Endodontics, Reciprocating movement, Permeability, R-pilot, Waveone Gold Glider

\section{INTRODUCCIÓN}

La conformación químico mecánica del sistema de conductos radiculares es un paso fundamental del tratamiento de Endodoncia(1). Las indicaciones de los fabricantes señalan que son instrumentos de uso único (2,3), sin embargo la condición social-económica de la región obligan al clínico a reusar su instrumental mecanizado, esto podría considerarse seguro siempre y cuando se ejecuten procedimientos basados en evidencia cientíica que demuestren que este comportamiento no implican la reducción de la eficacia ni riesgos de complicaciones.

El primer paso para la instrumentación es lo que se conoce como permeabilización, o glidepath, se puede definir como la creación de una trayectoria inicial que permita el acceso desde la entrada del canal radicular con un recorrido a través de toda su extensión en forma segura e ininterrumpida hasta el foramen apical (4).Para lo cual utilizamos limas mecanizadas que por las características de su aleación, los fabricantes proponen el uso único, para ser utilizada un máximo de 3 a 4 veces en un mismo molar de un mismo paciente (5). Se argumenta que el instrumento se desgasta y deforma, lo cual afecta su eficiencia de corte (6). Si se tiene en cuenta que su reutilización está acompañada de procesos de esterilización, se adicionan las alteraciones que este proceso pueda generar (7).

R-Pilot ${ }^{\circledR}$ es el primer instrumento mecanizado de NiTi para glidepath utilizado en un movimiento reciprocante (150/30) (8). Está hecho de aleación de M-Wire ${ }^{\circledR}$ y tiene una sección transversal en forma de S, un diámetro de punta de 0,125 mm y una conicidad constante del 4\%. M-Wire ${ }^{\circledR}$ corresponde a una aleación modificada del Nitinol(9). El tratamiento térmico patentado por NiTi M-Wire ${ }^{\circledR}$, tiene propiedades deseables superiores que los actuales instrumentos producidos de Nitinol508 y puede ser adoptado por otros diseños de limas para proveer avances significativos en el instrumental rotatorio.

El instrumento WaveOne Gold Glider ${ }^{\circledR}$ es una lima para realizar la permeabilidad del conducto radicular(10), tiene una sección transversal en forma de paralelogramo, tamaño individual (con un diámetro de punta de 15) y una conicidad que aumentan del 2\% a más del 6\% a lo largo de su parte activa. Su uso es único y utiliza exactamente los mismos ángulos desiguales bidireccionales en el sentido de las agujas del reloj; en sentido anti horario que las limas WaveOne Gold ${ }^{\circledR}$ (reciprocación en 170/50) (11).

Los instrumentos mecanizados de NiTi se desgastan o se fracturan sin signos previos de deformación que podría alarmar al clínico para su descarte, así que esto se considera una preocupación constante ya que la inspección visible no es un método fiable para evaluar los instrumentos de níquel-titanio usados (12).Los constantes esfuerzos llevan al material a un deterioro progresivo; con grietas debido a la concentración de tensiones, las cuales crecen hasta alcanzar un tamaño crítico, suficiente para la ruptura final, generalmente de forma brusca(13).

Las irregularidades superficiales forman puntos entrantes de concentración de tensión, que llevan a la formación de minúsculas grietas. De las características morfológicas y la cinemática de empleo de los instrumentos, dependen su capacidad de corte y su efectividad en mantener las características anatómicas de los conductos sin presentar deformaciones al momento de la conformación y limpieza (14).

Se pueden observar bajo los aumentos del microscopio electrónico de barrido, el cual muestra las diferentes formas de defectos superficiales como grietas que según la fatiga que recibió el instrumento pueden 
comenzar desde el núcleo del metal o desde la periferia, los cráteres que son socavamientos en la continuidad de la superficie, la presencia de barrido dentinario en el caso de limas con usos previos(15).

\section{MATERIALES Y MÉTODO}

Se realizó un estudio experimental comparativo in vitro con una muestra constituida por 10 instrumentos de cada tipo utilizados en canales curvos artificiales para tomar 600 fotografías en el microscopio electrónico de barrido. La confiabilidad de los canales de bloques de resina como modelo experimental aceptable para el análisis preliminar de la técnica de preparación endodóntica ha sido validada (16).Se observaron los defectos superficiales en los instrumentos nuevos, después 3, 6, 9 usos en canales artificiales y proceso de esterilización en autoclave a través del análisis de imágenes con SEM tomadas en aumentos de 500x. Los defectos superficiales que se analizaron en cada grupo de estudio fueron: micro-fisuras, deformación de estrías, cráteres, bordes romos, deformación de superficies cortantes, punta deformada, presencia de barrillo dentinario y fractura. La evaluación visual de las fotografías de las limas se realizó por tercios coronal, medio y apical por 3 especialistas en endodoncia cegados al tratamiento.

Se utilizaron cubos de acrílico estandarizados con conductos de $16 \mathrm{~mm}$ de longitud, curvatura de $45^{\circ}$, diámetro de 0,10 mm, se comprobó la permeabilidad de los mismos introduciendo una lima K10, hasta que se evidencie la punta del instrumento en el foramen apical artificial.

La muestra fue dividida aleatoriamente en dos grupos G1: R-pilot y G2: Wave One Gold Glider, cada una fue observada bajo el microscopio previo a la instrumentación, luego fueron insertadas en el motor inalámbrico, el cual presentó la opción para cada lima en particular y fueron usadas siguiendo el protocolo clínico propuesto por el fabricante, la observación se realizó cada tres cubos de acrílico simulando el número promedio de conductos que se encuentran en un molar.

Todos los canales fueron instrumentados por el mismo operador cegado al tratamiento y cada conducto fue irrigado con hipoclorito de sodio al 5.25\% mientras se lograba completar la longitud de trabajo de cada cubo, hasta terminar los tres cubos y luego cada lima se limpió con el siguiente protocolo: fueron sumergidas en jabón enzimático durante 20 minutos, luego se colocaron en el ultrasonido (Biowash STD ${ }^{\circledR}$ ) por 5 minutos. Las limas fueron cepilladas durante 30 segundos con cepillo de cerdas plásticas y chorro de agua, se secaron con toallas de papel desechables y se empacaron en bolsas individuales de polipropileno. Acto seguido, se llevaron a un ciclo de autoclave con el indicador químico a una temperatura de $121^{\circ} \mathrm{C}$ y 2 atmosferas de presión por 25 minutos y se secaron por 15 minutos.

Las limas utilizadas fueron llevadas al microscopio electrónico de barrido (JEOL, JSM 5610) y se fotografiaron por tercios: cervical, medio y apical. Estas mismas limas se reutilizaron en tres cubos nuevos y se las llevó nuevamente al proceso de autoclave para completar un total de seis conductos y dos ciclos de esterilización, y volver al microscopio electrónico de barrido para observar los defectos superficiales presentes en los mismos lugares del tercio apical, medio y coronal previamente evaluado.

Los criterios que se utilizaron para evaluar los instrumentos incluyeron la observación de la presencia o ausencia de defectos superficiales visibles: defectos superficiales microscópicos (Figura 1A), grietas, deformación plástica (geometría perdida o irregular del instrumento), las microfisuras sin una separación completa del instrumento, cráteres, (grandes fosas en la superficie); bordes romos (pérdida de la nitidez de los bordes de corte) y la presencia de restos de dentina.

Los defectos macroscópicos como fracturas completas (Figura 1B), que se definió como la separación del instrumento durante las pruebas.

\section{RESULTADOS}

Los datos obtenidos fueron tabulados después de la observación de las fotografías obtenidas con el SEM por tres endodoncistas calibrados y cegados al uso de las muestras, se procedió a unificar la información en una base de datos de un documento de Excel, después se realizó la distribución de los datos, la estadística descriptiva y la adherencia a la curva de normalidad por el test de ShapiroWilks. Los datos fueron analizados con los test de Kruskal-Wallis y Kolmogorov - Smirnov. (Tabla 1)

Cuando la lima es de la marca R-Pilot, se encontró solamente 1 deformación en promedio. Cuando es de la marca WaveOne Gold Glider, el promedio asciende a 2 deformaciones en total. (Tabla 2)

Cuando la lima tiene entre 0 y 3 usos, en promedio, mostró un solo defecto superficial. Pero, cuando asciende a 6 y 9 usos, el promedio de defectos observados sube a 2. (Tabla 3)

En promedio, se encontró 1.3 veces los bordes no cortantes y la deformación de estría. En 1.4 veces de promedio, se halló grietas. Y en 1.6 veces en promedio se observaron cráteres y micro-fisuras. (Tabla 4) 
Para las limas R-Pilot, en todos los casos se observó un promedio de detección ascendente conforme se aumenta su número de usos. Destaca la observación de cráteres en un promedio de 2 detecciones con 9 usos. Mientras que para las limas WaveOne Gold Glider, el promedio de observaciones de deformaciones es también creciente destacando las 2.5 veces que en promedio se detectaron micro-fisuras. (Tabla 5)

Para las limas R-Pilot, en todos los casos de deformaciones medidas, se observó un promedio mayor de fallos detectados en el tercio apical. Mientras que para las limas WaveOne Gold Glider, el promedio de observaciones de deformaciones hallado es también mayor en el tercio apical. Con excepción de los bordes no cortantes, siempre se registró un mayor promedio de deformaciones en el tercio apical para las limas WaveOne Gold Glider respecto a lo registrado para las limas R-Pilot. (Tabla 6)

Tanto en el caso de las limas R-Pilot como de las WaveOne Gold Glider, se registra un mayor promedio de deformaciones observadas conforme aumenta el uso de la lima.

Se pudo destacar que, en ambas marcas, el menor promedio de observaciones de fallos con 9 usos se da en el tercio coronal y el mayor promedio de fallos con 9 usos se registra en el tercio apical.

\section{DISCUSIÓN}

Lograr la permeabilidad del conducto radicular de forma segura y rápida constituye la llave del éxito en la instrumentación con limas mecanizadas de NiTi(17), para lo cual contamos actualmente con dos opciones en movimiento reciprocante: las limas R-pilot ${ }^{\circledR}$ y WaveOne Gold Glider ${ }^{\circledast}$

Los protocolos de las casas comerciales señalan que son limas de uso único (18), para reducir la fatiga del instrumento y la posibilidad de contaminación cruzada, sin embargo, se reutilizan en la práctica clínica por razones económicas, y actualmente no hay acuerdo en la literatura con respecto al número de reusos y la probabilidad de fractura de un instrumento (19).

Los primeros resultados del estudio con estas limas sugieren que R-Pilot ${ }^{\circledR}$ tiene una mayor resistencia a la fractura por torsión que WaveOne Gold Glider ${ }^{\circledR}$, el mayor diámetro de la sección transversal podría influir en este resultado. Özyrüek et al. (2018) demostraron que WaveOne Gold Glider ${ }^{\circledR}$ tenía una mayor resistencia a la flexión en comparación con los instrumentos R-Pilot ${ }^{\circledR}$

WaveOne Gold Glider ${ }^{\circledR}$ incorpora una nueva aleación NiTi, que se ha sometido a un tratamiento termo mecánico que tiene mayor flexibilidad en comparación con la aleación M-Wire ${ }^{\circledR}$ (22), lo que puede aumentar la capacidad de deformación de este instrumento (20). En este estudio se observó en las limas WaveOne Gold Glider ${ }^{\circledR}$ la presencia de alteraciones notables en las espirales de corte (deformación plástica) y mayor incidencia de punta deformada probablemente debido a la mayor ductilidad del metal y esto podría ser útil durante el uso clínico, ya que nos podría advertir de una futura fractura del instrumento.

Los defectos más frecuentes observados en las limas R-pilot ${ }^{\oplus}$ fueron los bordes romos y los cráteres, presentó una estructura más uniforme a través de su superficie y menos defectos superficiales en comparación con WaveOne Gold Glider ${ }^{\circledR}$ incluso después de los reúsos, por lo que la fractura puede ocurrir sin ninguna deformación previa.

En este estudio se pudo verificar que las microfisuras y los cráteres son totalmente independientes del tiempo de uso de las limas, ya que se encontraron inclusive antes de ser utilizadas (21). Por el contrario, al hablar de deformación de estrías y bordes romos podemos afirmar que entre más usos más se presentaban, es decir, es dependiente del número de reúsos (22). Los cráteres se observaron en ambos sistemas, sin embargo, se puede observar una diferencia significativa en las limas WaveOne Gold Glider ${ }^{\circledR}$ después del sexto uso.

Para determinar un número de reúsos de estas limas reciprocantes, se requiere una investigación adicional y verificar la resistencia a la fatiga cíclica o torsional después de usos múltiples, y no solo la presencia de defectos superficiales, ya que este estudio también encontró alteraciones en la mayoría de los instrumentos nuevos, y a medida que aumentaba el número de usos, el proceso de esterilización y la fatiga del material, se observaba como se afectaba la microestructura de las limas de diámetro pequeño con bastante facilidad, probablemente como resultado del estrés en la sección transversal relativamente pequeña en canales radiculares estrechos y curvos(23).

Las limas R-pilot ${ }^{\circledR}$ tuvieron una resistencia superior al inicio de grietas por fatiga, lo que refleja potencialmente la reorientación de las variantes martensíticas resultante de la simetría más baja de la estructura cristalina monoclínica de martensita que la estructura cristalina cúbica de austenita. Los defectos más prevalentes fueron bordes romos y cráteres que probablemente son resultado del uso de una sustancia química (5.25\% de NaOCl) en la preparación del canal. 
Cunha y col. (24) estudiaron el comportamiento de los instrumentos recíprocos después de un solo uso, y aun así sus resultados de tasa de fractura fueron comparables con los nuestros en los que cada instrumento se usó para la preparación de 3 dientes posteriores. La experiencia clínica que se obtuvo durante el período de este estudio indica que los instrumentos evaluados se pueden usar de manera segura en hasta 3 casos clínicos de tratamiento endodóntico en dientes posteriores

Plotino y col. (2012) (25) informaron que los ciclos repetidos (10 ciclos) de esterilización en autoclave $\left(134^{\circ} \mathrm{C}\right.$ durante $\left.35 \mathrm{~min}\right)$ no influyeron en las propiedades mecánicas de los instrumentos NiTi. En un estudio clínico a gran escala, muchos factores clínicos, como el tipo de dientes, la morfología del conducto radicular (curvatura, longitud y anchura) y la variabilidad del operador, son bastante difíciles de controlar.
En nuestro trabajo solo dos instrumentos experimentan fractura. La limas R-pilot después de preparar 9 canales las fotomicrografías SEM mostraron que las grietas por fatiga en este archivo estaban presentes después del primer uso y la WaveOne Gold Glider ${ }^{\circledR}$ después de 6 canales. Todos los instrumentos que experimentaron fractura mostraron una rotura brusca sin ningún defecto visible acompañante.

\section{CONCLUSIONES}

WaveOne Gold Glider presentó mayores defectos superficiales después de la instrumentación de 9 canales curvos artificiales y 3 ciclos de esterilización en comparación las limas R-pilot.

Según los resultados de este ensayo realizado en canales curvos simulados en bloques de resina, es posible reutilizar los instrumentos mecanizados de permeabilidad tomando en cuenta su curvatura y ciclos de esterilización, lamentablemente no se puede prever signos visibles de deformación plástica que puedan conducir a la fractura del instrumento. Las limas R-Pilot presentaron menos defectos superficiales microscópicos, pero tuvieron una fractura inesperada, lo cual clínicamente podría ser perjudicial ya que no se pudo predecir.

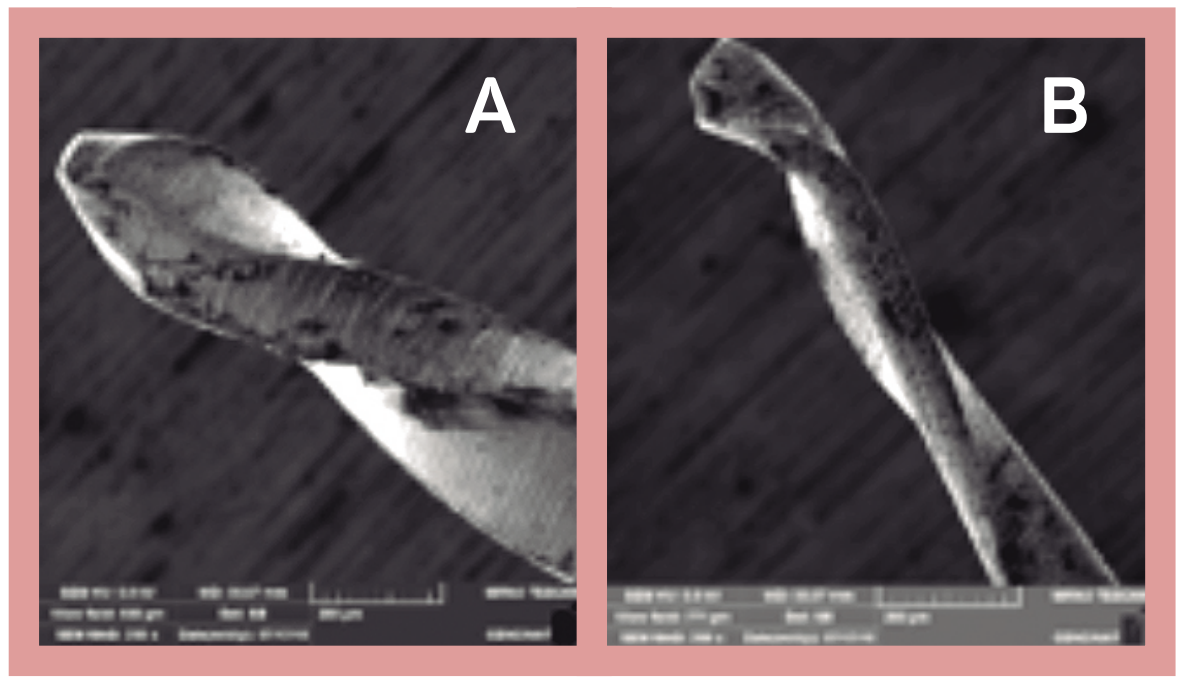

Figura 1:

A) Defecto microscópico B) Defecto macroscópico.

Tabla 1:

Test kruskal-wallis entre los grupos y el número de usos

\begin{tabular}{|l|r|r|r|r|r|r|r|r|}
\hline & \multicolumn{2}{|c|}{ CON O USOS } & \multicolumn{2}{c|}{ CON 3 USOS } & \multicolumn{2}{c|}{ CON 6 USOS } & \multicolumn{2}{c|}{ CON 9 USOS } \\
\hline & N & RANK SUM & N & RANK SUM & N & RANK SUM & N & RANK SUM \\
\hline R-P & 75 & 5521,5 & 75 & 5350,5 & 75 & 5230,5 & 75 & 5149 \\
\hline WOGG & 75 & 5803,5 & 75 & 5974,5 & 75 & 6094,5 & 75 & 6176 \\
\hline CHI-CUADRADO: & & 0,413 & & 1,608 & & 3,08 & \\
\hline P-VALUE: & & $\mathbf{0 , 5 2 0 5}$ & & $\mathbf{0 , 2 0 4 7}$ & & $\mathbf{0 , 0 7 9 3}$ & \\
\hline
\end{tabular}




\begin{tabular}{|c|c|c|}
\hline TIPO & MEDIA & DESVIACIÓN ESTÁNDAR \\
\hline R-PILOT & 1,266667 & 1,371797 \\
\hline WAVEONE GOLD GLIDER & 1,586667 & 1,391261 \\
\hline$N^{\circ}$ DE USOS & MEDIA & DESVIACIÓN ESTÁNDAR \\
\hline 0 & 0,74 & 1,166823 \\
\hline 3 & 1,326667 & 1,368371 \\
\hline 6 & 1,633333 & 1,372876 \\
\hline 9 & 2,006667 & 1,333596 \\
\hline DEFECTOS & MEDIA & DESVIACIÓN ESTÁNDAR \\
\hline BORDES NO CORTANTES & 1,283333 & 1,373111 \\
\hline DEFORMACIÓN DE ESTRÍA & 1,275 & 1,377871 \\
\hline CRÁTERES & 1,591667 & 1,423074 \\
\hline GRIETAS & 1,383333 & 1,342163 \\
\hline MICRO-FISURAS & 1,6 & 1,416588 \\
\hline
\end{tabular}

TABLA 2.

Defectos superficiales en relación con la lima evaluada

\section{TABLA 3.}

Defectos superficiales en relación con número de usos

\section{TABLA 4.}

Defectos superficiales en la limas de glide-path

\begin{tabular}{|c|c|c|c|c|c|}
\hline \multicolumn{6}{|c|}{ BORDES NO CORTANTES } \\
\hline & & 0 & 3 & 6 & 9 \\
\hline \multirow{2}{*}{ R-PILOT } & Media & 0,5333334 & 1,2 & 1,333333 & 1,533333 \\
\hline & Desviación estándar & 1,125463 & 1,373213 & 1,345185 & 1,45733 \\
\hline \multirow{2}{*}{$\begin{array}{l}\text { WAVEONE GOLD } \\
\text { GLIDER }\end{array}$} & Media & 0,6666667 & 1,266667 & 1,533333 & 2,2 \\
\hline & Desviación estándar & 1,112697 & 1,334523 & 1,407463 & 1,373213 \\
\hline \multicolumn{6}{|c|}{ DEFORMACIÓN DE ESTRIAA } \\
\hline & & 0 & 3 & 6 & 9 \\
\hline \multirow{2}{*}{ R-PILOT } & Media & 0,3333333 & 0,8 & 1,4 & 1,866667 \\
\hline & Desviación estándar & 0,8164966 & 1,146423 & 1,454058 & 1,407463 \\
\hline \multirow{2}{*}{$\begin{array}{l}\text { WAVEONE GOLD } \\
\text { GLIDER }\end{array}$} & Media & 0,6666667 & 1,333333 & 1,733333 & 2,066667 \\
\hline & Desviación estándar & 1,112697 & 1,447494 & 1,387015 & 1,387015 \\
\hline \multicolumn{6}{|c|}{ CRÁTERES } \\
\hline & & 0 & 3 & 6 & 9 \\
\hline \multirow{2}{*}{ R-PILOT } & Media & 0,8 & 1,533333 & 1,733333 & 2,066667 \\
\hline & Desviación estándar & 1,264911 & 1,407463 & 1,486447 & 1,334523 \\
\hline \multirow{2}{*}{$\begin{array}{l}\text { WAVEONE GOLD } \\
\text { GLIDER }\end{array}$} & Media & 0,7333333 & 1,533333 & 2 & 2,333333 \\
\hline & Desviación estándar & 1,279881 & 1,45733 & 1,36277 & 1,234427 \\
\hline \multicolumn{6}{|c|}{ GRIETAS } \\
\hline & & 0 & 3 & 6 & 9 \\
\hline \multirow{2}{*}{ R-PILOT } & Media & 0,7333333 & 1,2 & 1,266667 & 1,6 \\
\hline & Desviación estándar & 1,162919 & 1,373213 & 1,387015 & 1,404076 \\
\hline \multirow{2}{*}{$\begin{array}{l}\text { WAVEONE GOLD } \\
\text { GLIDER }\end{array}$} & Media & 0,8666667 & 1,466667 & 1,8 & 2,133333 \\
\hline & Desviación estándar & 1,125463 & 1,407463 & 1,320173 & 1,245946 \\
\hline
\end{tabular}




\begin{tabular}{|c|c|c|c|c|}
\hline \multicolumn{5}{|c|}{ BORDES NO CORTANTES } \\
\hline & & CORONAL & MEDIO & APICAL \\
\hline \multirow{2}{*}{ R-PILOT } & Media & 0,25 & 1 & 2,2 \\
\hline & Desviación estándar & 0,7163504 & 1,213954 & 1,281447 \\
\hline \multirow{2}{*}{$\begin{array}{l}\text { WAVEONE GOLD } \\
\text { GLIDER }\end{array}$} & Media & 0,8 & 1,3 & 2,15 \\
\hline & Desviación estándar & 1,239694 & 1,380313 & 1,268028 \\
\hline \multicolumn{5}{|c|}{ DEFORMACIÓN DE ESTRÍA } \\
\hline & & CORONAL & MEDIO & APICAL \\
\hline \multirow{2}{*}{ R-PILOT } & Media & 0,5 & 1,15 & 1,65 \\
\hline & Desviación estándar & 1,051315 & 1,268028 & 1,460894 \\
\hline \multirow{2}{*}{$\begin{array}{l}\text { WAVEONE GOLD } \\
\text { GLIDER }\end{array}$} & Media & 0,85 & 1,35 & 2,15 \\
\hline & Desviación estándar & 1,225819 & 1,424411 & 1,308877 \\
\hline \multicolumn{5}{|c|}{ CRÁTERES } \\
\hline & & CORONAL & MEDIO & APICAL \\
\hline \multirow{2}{*}{ R-PILOT } & Media & 1,05 & 1,6 & 1,95 \\
\hline & Desviación estándar & 1,356272 & 1,429022 & 1,394538 \\
\hline \multirow{2}{*}{$\begin{array}{l}\text { WAVEONE GOLD } \\
\text { GLIDER }\end{array}$} & Media & 1,25 & 1,5 & 2,2 \\
\hline & Desviación estándar & 1,482352 & 1,468977 & 1,239694 \\
\hline \multicolumn{5}{|c|}{ GRIETAS } \\
\hline & & CORONAL & MEDIO & APICAL \\
\hline \multirow{2}{*}{ R-PILOT } & Media & 0,4 & 1,3 & 1,9 \\
\hline & Desviación estándar & 0,8207827 & 1,380313 & 1,333772 \\
\hline \multirow{2}{*}{$\begin{array}{l}\text { WAVEONE GOLD } \\
\text { GLIDER }\end{array}$} & Media & 1,1 & 1,45 & 2,15 \\
\hline & Desviación estándar & 1,209611 & 1,394538 & 1,225819 \\
\hline \multicolumn{5}{|c|}{ GRIETAS } \\
\hline & & CORONAL & MEDIO & APICAL \\
\hline \multirow{2}{*}{ R-PILOT } & Media & 0,7 & 1,3 & 2,05 \\
\hline & Desviación estándar & 1,174286 & 1,49032 & 1,276302 \\
\hline \multirow{2}{*}{$\begin{array}{l}\text { WAVEONE GOLD } \\
\text { GLIDER }\end{array}$} & Media & 1,45 & 1,75 & 2,35 \\
\hline & Desviación estándar & 1,431782 & 1,409554 & 1,225819 \\
\hline
\end{tabular}

\section{TABLA 6 .}

Defectos microscópicos

y tercio de medición.

\section{REFERENCIAS BIBLIOGRÁFICAS}

1. Schilder H. Cleaning and shaping the root canal. Dental clinics of North America. 1974;18(2):269-96. Epub 1974/04/01.

2. Walia HM, Brantley WA, Gerstein H. An initial investigation of the bending and torsional properties of Nitinol root canal files. Journal of endodontics. 1988;14(7):346-51. Epub 1988/07/01.

3. Berutti, E.; Cantatore, G.; Castellucci, A.; Chiandussi, G.; Pera, F.; Migliaretti, G. \&Pasqualini, D. Use of nickel-titanium rotary PathFile to create the glide path: comparison with manual preflaring in simulated root canals. J. Endod., 35(3):408-12, 2009.

4. Türker, S. A. \&Uzunoglu, E. Apical root canal transportation of different pathfinding systems and their effects on shaping ability of ProTaper Next. J. Clin. Exp. Dent., 7(3):e392-5, 2015

5. Sattapan B, Nervo GJ, Palamara JE, Messer HH. Defects in rotary nickel-titanium files after clinical use. Journal of endodontics. 2000;26(3):161-5. Epub 2001/02/24. 6. Baek SH, Lee CJ, Versluis A, Kim BM, Lee W, Kim HC. Comparison of torsional stiffness of nickel-titanium rotary files with different geometric characteristics. Journal of endodontics. 2011;37(9):1283-6. Epub 2011/08/19

7. Pinheiro E, Gomes B, Ferraz C, Sousa E, Teixeira F, Souza F. Microorganisms from canals of root-filled teeth with periapical lesions. IntEndod J. 2003; 36(1): 1-11.

8. Todd P, Whitney S, Walker S, Friedman S. Microbial contamination of endodontic files received from the manufacturer. J Endod. 2006; 32(7): 649-51.

9. Linsuwanont $\mathrm{P}$, Parashos $\mathrm{P}$, Messer $\mathrm{H}$. Cleaning of rotary nickel-titanium endodontic instruments. IntEndod J. 2001; 37: 19-28.

10. Johnson M, Primack P, Loushine R, Craft D. Cleaning of endodontic files, part I: the effect of bioburden on the sterilization of endodontic files. J Endod. 1997; 23(1): 32-4. 11. Parashos $P$, Linsuwanont $P$, Messer $\mathrm{HH}$. A cleaning protocol for rotary nickel-titanium endodontic instruments. AustDent J. 2004; 49(1): 20-7.

12. Smith A, Lettersa S, Langeb A, Perrettc D, McHugha S, Bagga J. Residual protein levels on reprocessed dental instruments. J Hosplnfect. 2005; 61: 237-41.

13. Sonntag D, Ove A. Effect of prion decontamination protocols on nickel-titanium rotary surfaces. J Endod. 2007; 33(4): 442-6.

14. Venkatasubramanian $R$, Jayanthi, Das U, Bhatnagar S. Comparison of the effectiveness of sterilizing endodontic files by 4 different methods: An in vitro study. JournallndianSocietyof Pedodonticsand PreventiveDent. 2010; 28(1): 2-5.
15. Lemmer K, Mielke K, Georg P, Beekes M.

Decontamination of surgical instruments from prion proteins: in vitro studies on the detachment, destabilization and degradation of PrPSc bound to steel surfaces. J Gen Virol. 2004; 85: 3805-16.

16. Cheung GS, Shen Y, Darvell BW. Does electropolishing improve the low-cycle fatigue behavior of a nickel-titanium rotary instrument in hypochlorite? J Endod 2007;33:1217 17. Sattapan B, Nervo GJ, Palamara JE, Messer HH. Defects in rotary nickel-titanium files after clinical use. J Endod 2000;26:161-5.

18. Martín B, Zelada G, Varela P, et al. Factors influencing the fracture of nickel-titanium rotary instruments. International Endodontic Journal 2003;36:262- 6

19. Parashos P, Gordon I, Messer HH. Factors influencing defects of rotary nickeltitanium endodontic instruments after clinical use. J Endod 2004;30:722-5 20. Rapisarda E, Bonaccorso A, Tripi TR, Fragalk I, Condorelli GG (2000) The effect of surface treatments of nickel-titanium files on wear and cutting efficiency. Oral Surgery, Oral Medicine,Oral Pathology,Oral Radiology and Endodontology 89, 363-8

21. Yao JH, Schwartz SA, Beeson TJ.Cyclic fatigue of three types of rotary nickel-titanium files in a dynamic model. Journal of Endodontics2006;32, 55-7

22. Valois, C. R. A. Silva, L. P., \&Azevedo, R. B. Atomic Force Microscopy Study of Stainless-Steel and Nickel-Titanium Files. Journal of Endodontics 2005; 31(12), 882-885. 23. Sattapan, B., Nervo, G. J., Palamara, J. E., \& Messer, H. $H$. Defects in rotary nickel-titanium files after clinical use. Journal of Endodontics 2000; 26(3), 161-165

24. Sanches-Cunha R. Evaluation of the incidence of reciprocal wave one file separation: a prospective clinical study Journal of Endodontics. 2014; 40 (7): 922-924 25. Plotino G, Grande NM, Cordaro M, Testarelli L, Gambarini G. A review of cyclic fatigue testing of nickel-titanium rotary instruments. Journal of Endodontics. 2009:35(11):1469-76. 